Ann. Geophys., 36, 337-347, 2018

https://doi.org/10.5194/angeo-36-337-2018

(C) Author(s) 2018. This work is distributed under

the Creative Commons Attribution 3.0 License.

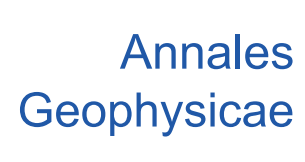

(c) (i)

\title{
On the relevance of source effects in geomagnetic pulsations for induction soundings
}

\author{
Anne Neska ${ }^{1}$, Jan Tadeusz Reda ${ }^{1}$, Mariusz Leszek Neska ${ }^{1}$, and Yuri Petrovich Sumaruk ${ }^{2}$ \\ ${ }^{1}$ Institute of Geophysics, Polish Academy of Sciences, ul. Ks. Janusza 64, 01-452 Warsaw, Poland \\ ${ }^{2}$ Institute of Geophysics of the National Academy of Sciences of the Ukraine, Kiev, Ukraine
}

Correspondence: Anne Neska (anne@igf.edu.pl)

Received: 3 March 2017 - Revised: 13 January 2018 - Accepted: 24 January 2018 - Published: 7 March 2018

\begin{abstract}
This study is an attempt to close a gap between recent research on geomagnetic pulsations and their usage as source signals in electromagnetic induction soundings (i.e., magnetotellurics, geomagnetic depth sounding, and magnetovariational sounding). The plane-wave assumption as a precondition for the proper performance of these methods is partly violated by the local nature of field line resonances which cause a considerable portion of pulsations at mid latitudes. It is demonstrated that and explained why in spite of this, the application of remote reference stations in quasiglobal distances for the suppression of local correlated-noise effects in induction arrows is possible in the geomagnetic pulsation range. The important role of upstream waves and of the magnetic equatorial region for such applications is emphasized. Furthermore, the principal difference between application of reference stations for local transfer functions (which result in sounding curves and induction arrows) and for inter-station transfer functions is considered. The preconditions for the latter are much stricter than for the former. Hence a failure to estimate an inter-station transfer function to be interpreted in terms of electromagnetic induction, e.g., because of field line resonances, does not necessarily prohibit use of the station pair for a remote reference estimation of the impedance tensor.
\end{abstract}

Keywords. Geomagnetism and paleomagnetism (geomagnetic induction) - ionosphere (ionosphere-atmosphere interactions) - magnetospheric physics (general or miscellaneous)

\section{Introduction}

In this section we motivate our study by adducting a number of contradictions and ambiguities that are encountered both within the literature on electromagnetic induction soundings and between literature and empirical findings. These are demonstrated on data which will be documented in the Data section and which have been processed by methods introduced in the Method section. The contradictions will be solved in the light of recent research in the Results and discussion section.

Here we refer to the magnetotelluric (MT), magnetovariational (MV), and geomagnetic depth sounding (GDS) methods as induction methods. The aim of such induction soundings is to obtain a model of the distribution of electrical resistivity in the subsurface of the solid earth which enables a geologic-tectonic interpretation. Transfer functions between electromagnetic field components measured at the surface or on the sea floor constitute the input data for such a modeling. The precondition for the proper functioning of such a modeling is that transfer functions depend only on electromagnetic induction in the subsurface. This means in particular that the geometry of the source field, i.e., of the incident electromagnetic wave which drives that induction, can be neglected. This condition is referred to as the plane-wave assumption. By this term it is understood that the incident wave field is homogeneous over a large area on the surface. "Large" means here large compared to the skin depth that characterizes how deep this wave penetrates into the solid earth. This is an elementary consequence of a basic equation in electrodynamics - the telegrapher's equation which describes the damped propagation of an electromagnetic wave in an electrically conducting medium. 
The source field in the range of long-period magnetotellurics (LMT, comprising periods from 10 to $10000 \mathrm{~s}$ ) is constituted by the geomagnetic pulsations and variations sensu stricto, e.g., of geomagnetic storm, substorm, and bay types. All of them take their energy from the solar wind, and they are explained by either fluctuations in the solar wind or its interaction with the terrestrial magnetosphere. In this study we focus on the pulsation range that is limited to periods $<600$ s and characterized by frequently occurring sinusoidal signals. For this range textbooks of MT and other works introducing source signals for soundings (e.g., Bahr and Simpson, 2005; Houpt, 2008; Viljanen, 2012) list field line resonances, waveguides, and cavity modes as generation mechanisms. Whereas it is accepted that in general LMT sources meet the plane-wave assumption, two exceptions to this rule are highlighted in the literature, which will be considered in the following.

The first exception concerns certain regions in which all LMT source signals are inhomogeneous according to, e.g., Bahr and Simpson (2005). These regions are situated beneath a polar or the equatorial electrojet. We focus on the latter here because this region is more important for our study (and because the high latitudes are rather different in terms of geomagnetic pulsations). The equatorial electrojet is (similar to the Sq variation at mid latitudes) a part of the current system developing in the dayside ionosphere due to solar radiation; i.e., it has a diurnal periodicity at a given place at the surface. The literature (Vassal et al., 1998; Bahr and Simpson, 2005; Padilha et al., 2017) warns of using data recorded during local daytime for determining transfer functions in equatorial regions and advises one to limit their estimation to a basis of local nighttime data. Unfortunately, it is not explained how an ionospheric current system with diurnal periodicity can make LMT source signals (which have much shorter periods and are generated at a much larger distance from the surface) inhomogeneous, and why this happens in electrojet and not in Sq regions. In other words, the impression is created that the equatorial electrojet is causal for anomalous properties of daytime LMT sources in the same region, although the mechanism of cause and effect is not revealed. The statement that the diurnal electromagnetic signals originating from that electrojet itself are not appropriate for sounding (Vassal et al., 1998) does not contribute to an answer to this question given the fact that the $\mathrm{Sq}$ variation cannot be used in the classical induction methods either. As a result, a large region of the earth is to some degree "distrusted" in terms of induction methods without thorough reasoning.

The second exception to the rule that LMT sources produce plane incident waves concerns all pulsations generated by field line resonances. Field line resonances are an everyday phenomenon. They are standing magnetohydrodynamic waves (Alfvenic shear waves) propagating along the main field lines through the plasmasphere and ionosphere. Their periods match the length of the given field line (which is characterized by the $L$-value, McIlwain, 1961) and therefore depend significantly on geomagnetic latitude. For geomagnetic mid latitudes most of them are in the pc3 range (10$45 \mathrm{~s}$ ). At the footpoints of the field lines ionospheric currents are driven which radiate energy in the form of electromagnetic waves to the ground where they are recognized as pulsations (McPherron, 2005). Given the situation that in principle every field line is able to generate its own electromagnetic wave with a latitude-dependent period, it is quite clear that the wave fronts observed on ground will be inhomogeneous rather than plane. This will be the case even if a set of neighboring field lines is excited synchronously (coupled resonances, Cladis, 1971), and inhomogeneity will be most striking when observed at two longitude-aligned stations in a distance greater than the one between footpoints of two field lines. This problem is pointed out in textbooks (Viljanen, 2012) and is the objective of several research articles (Egbert et al., 2000; Pilipenko and Fedorov, 1993). The latter show a synthetic apparent resistivity curve with distortions caused by resonant features in the source field. The former address violations of the plane-wave assumption, which have been noticed in inter-station transfer functions and which changed with a diurnal periodicity. The identification of field line resonances as the cause was possible due to an additional large eigenvalue in the spectral density matrix of the measured field components. It is pointed out in the same work that such an additional large eigenvalue in the applied processing method is indicative of DC railway noise as well. DC railway noise is an obviously harmful anthropogenic violation of the plane-wave assumption that often causes bad distortions in transfer functions (Schäfer et al., 2011; Padua et al., 2002; Larsen et al., 1996). A further proof of the local, inhomogeneous character of pulsations seems to be their coherence. Calculated for March 2013, it is only between "neighboring" observatories (BEL-KIV sites in Poland and Ukraine, Europe) greater than 0.5 , but is close to zero for most distant pairings and periods (Table 1). Given these findings one may honestly wonder whether induction soundings in the pulsation range are possible at all.

However, this pessimistic view is counterbalanced by the empirical situation. MT in the pulsation range is done routinely and as a matter of course, and neither are hints on possibly source-related problems in this range a ubiquitous phenomenon in the literature on regional MT nor can the authors of this study confirm such problems from their own experience. A further argument comes from the remote-reference (RR) technique, which utilizes data of a second site to stabilize and correct local transfer functions of some site in MT and GDS. This technique relies on coherence of data between both sites, and it turns out that its successful application is possible even for distant reference sites. Campanyà et al. (2014) report on such an application of a German observatory to data from the Pyrenees and even from Morocco (northern Africa). Figure 1 shows how a heavy distortion by DC railway noise in a GDS sounding at a Polish observatory (BEL, treated in detail in Neska et al., 2013) is corrected by 
Table 1. Coherences between $H$ components of observatories in "neighborhood" (KIV-BEL) and "inter-continental" (all others) distances. See Sect. 2 for their locations. Data from the entire March 2013.

\begin{tabular}{lrrrrrr}
\hline Period (s) & 22 & 44 & 88 & 176 & 352 & distance \\
\hline KIV-BEL & 0.36 & 0.52 & 0.58 & 0.64 & 0.77 & $700 \mathrm{~km}$ \\
KIV-MBO & 0.01 & 0.07 & 0.16 & 0.35 & 0.31 & $5800 \mathrm{~km}$ \\
MBO-BOU & 0.06 & 0.04 & 0.04 & 0.03 & 0.13 & $8900 \mathrm{~km}$ \\
KIV-BOU & 0.11 & 0.01 & 0.02 & 0.07 & 0.02 & $9000 \mathrm{~km}$
\end{tabular}

means of a number of different reference sites. It is shown that reference sites from other continents yield results basically equal (even if not as smooth) to a classical reference site from the same region. This works under the condition of a sufficient data amount, where "sufficient" means a multiple of the data amount necessary for an everyday LMT sounding (ca. 3 months in contrast to 2-3 weeks). One of these references (MBO) is situated in Senegal in the region of the magnetic Equator.

Another contradiction is demonstrated in Fig. 2. The upper part (a) shows an inter-station transfer function between two Polish sites which completely confirms the objections in Egbert et al. (2000). Its behavior at periods $<100 \mathrm{~s}$ is not a small deviation varying smoothly over period from the unity matrix, but makes no sense at all in the paradigm of induction and would be treated as failure. Interestingly, the magnetotelluric transfer functions based on data from the same time (b of one of the sites, $c$ of both sites) are not unusual, problematic or suspicious in this period range. Thus we finish this section by posing two questions. First, why does RR with distant reference sites work in spite of low coherences between both sites? And second, why does a violation of the plane-wave assumption lead to a failure in estimating a reasonable inter-station transfer function, but is not an obstacle to determining a magnetotelluric transfer function?

\section{Data}

In this study selected data from the International Real-time Magnetic Observatory Network (INTERMAGNET, www. intermagnet.org) have been used, which consists of ca. 100 observatories all over the globe. Some years ago INTERMAGNET introduced the possibility of publishing and accessing geomagnetic variation data with an increased sampling rate of $1 \mathrm{~Hz}$ additionally (Turbitt et al., 2013), whereas the shortest sampling interval that could be published prior to that was $1 \mathrm{~min}$ (Jankowski and Sucksdorff, 1996). These $1 \mathrm{~s}$ data offer the chance to investigate almost the whole pulsation range (with the exception of pc1 and pi1) practically in a world-wide manner.

We included data of five INTERMAGNET observatories in our analysis. KIV and BEL are situated in Europe, BOU
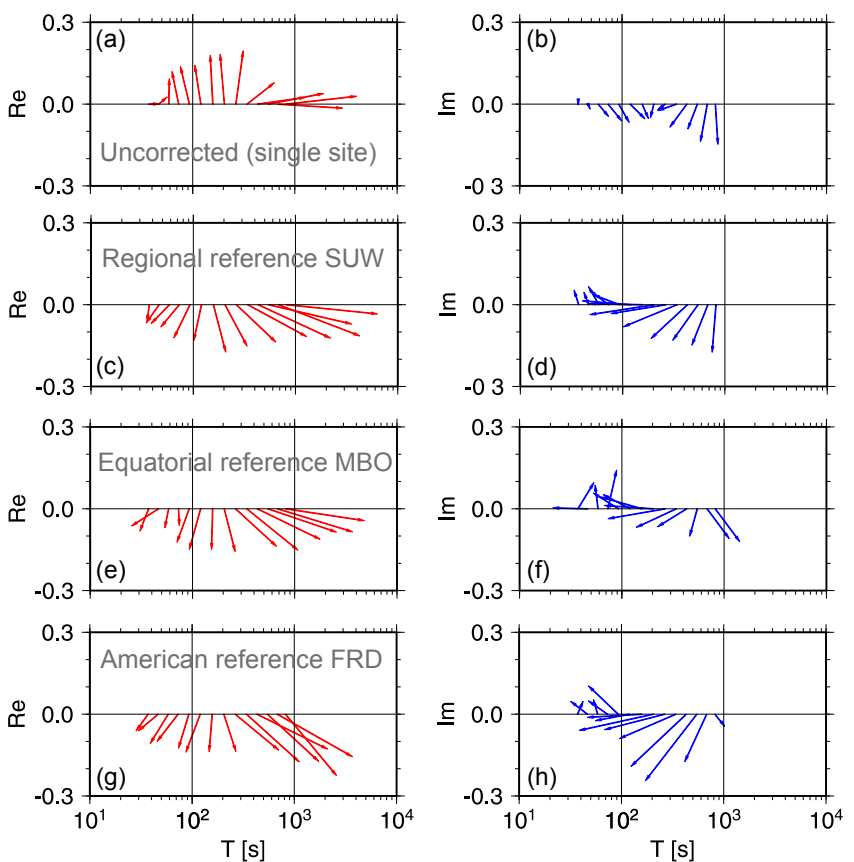

Figure 1. Real (a, c, e, g) and imaginary (b, d, f, h) induction arrows for Belsk observatory. The north-pointing real arrows in the single-site processed upper panel are an effect of distortion by DC railway noise. This effect vanishes if data are processed with the remote reference method (three lower panels). Very far reference sites (MBO, Senegal, and FRD, US) are similarly helpful to a classical regional one (SUW) if the data amount is sufficiently high (15 days for both upper panels, 101 days with MBO, 85 days with FRD).

and FRD in North America, and MBO in Africa at the geomagnetic Equator. Further data have been taken from ODE (an observatory in the Ukraine), SUW (a permanent variometer station in Poland), and GRB (a long-term magnetotelluric station in Poland from a running project). Table 2 introduces corrected geomagnetic coordinates (CGM from omniweb.gsfc.nasa.gov for 2013) and $L$-values (ibid.) of these sites. They are mapped in Fig. 3. For all sites the north $(X)$ and east $(Y)$ components of the magnetic variation data have been used. Because of the GDS study the vertical magnetic component $(Z)$ has been included for BEL. The GRB magnetotelluric site also comprises $1 \mathrm{~s}$ records of both perpendicular horizontal electric field components.

\section{Method}

\subsection{Coherence analysis}

The INTERMAGNET geomagnetic variation data have been rotated from the geographic $(X, Y, Z)$ to geomagnetic $(H, D, Z)$ coordinate systems. Spectral values have been produced by means of the wavelet transform, a tool that is often applied in geomagnetic and related studies, e.g., by Balasis et al. (2005), Heilig et al. (2007), Garcia and Jones (2008), 

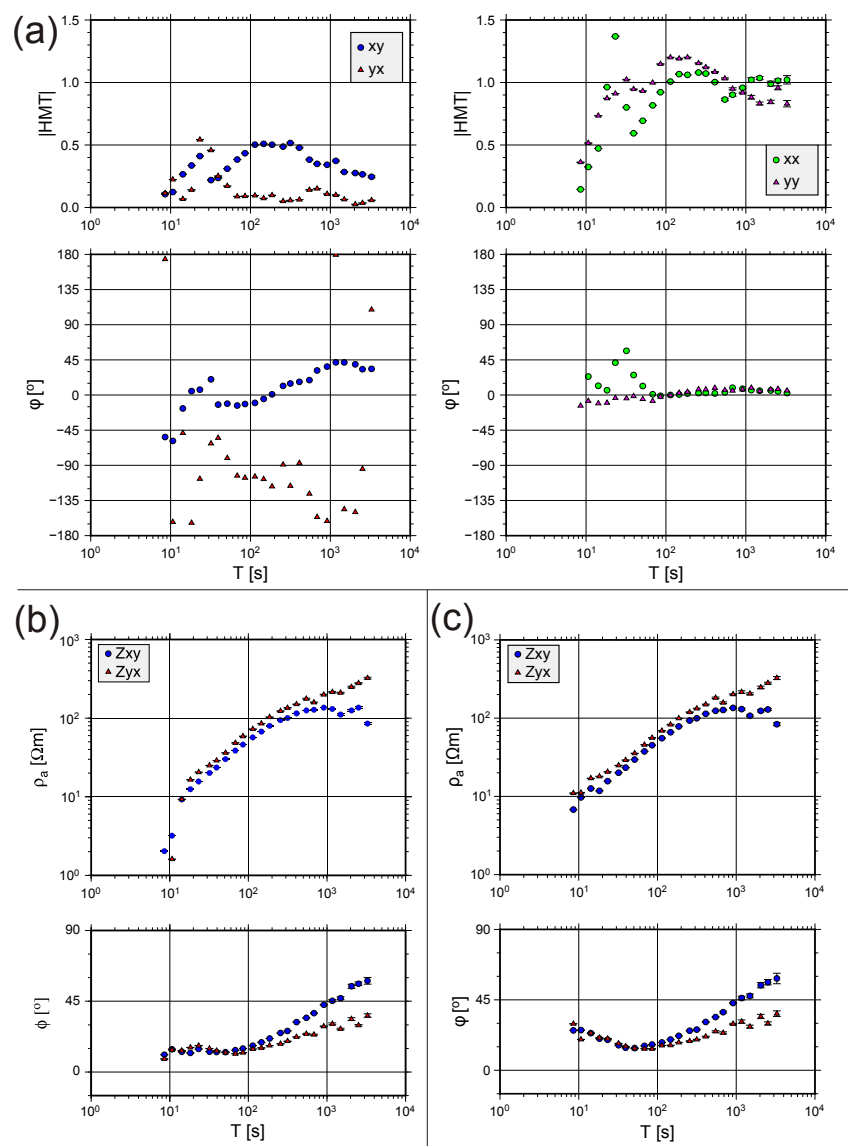

Figure 2. The inter-station transfer function between sites GRB and SUW (a) behaves erratically (from an induction point of view) for periods below $100 \mathrm{~s}$. Sounding curves for GRB from the same data (amount 5 days, (b) single-site, (c) remote-reference with SUW, only off-diagonal elements shown) in this period range do not show unusual features.

and Chernogor (2009). Thereby a signal depending on time $t$, e.g., $H(t)$, is subjected to a convolution with a wavelet function $\Psi$ (an asterisk ${ }^{*}$ denotes the complex conjugate):

$W_{\mathrm{H}}(a, b)=\frac{1}{\sqrt{|a|}} \int_{-\infty}^{\infty} H(t) \Psi^{*}\left(\frac{t-b}{a}\right) \mathrm{d} t$.

As $\Psi$ we used the Morlet wavelet (as done by all authors cited above) which has a shape matching pulsations:

$$
\begin{gathered}
\Psi_{\text {Morlet }}(t)=\pi^{1 / 4} \exp \left(-\frac{t^{2}}{2}\right) \exp \left(i \omega_{0} t\right), \\
\omega_{0}=\pi \sqrt{2 / \ln (2)},
\end{gathered}
$$

where $\omega_{0}$ is the dimensionless frequency parameter of the Morlet wavelet (Balasis et al., 2013). The resulting wavelet coefficients $W_{\mathrm{H}}$ are functions of $a$ and $b$, where $a$ describes the dilatation of the wavelet pattern. It represents the frequency about which the given coefficient yields information.
Table 2. Locations, geomagnetic coordinates (CGM), and $L$-values of the used sites.

\begin{tabular}{lrrr}
\hline $\begin{array}{l}\text { Site } \\
\text { (abbreviation), } \\
\text { country }\end{array}$ & $\begin{array}{r}\text { Geomagnetic } \\
\text { latitude } \\
\text { (CGM) }\end{array}$ & $\begin{array}{r}\text { Geomagnetic } \\
\text { longitude } \\
\text { (CGM) }\end{array}$ & $L$-value \\
\hline $\begin{array}{l}\text { Belsk (BEL), } \\
\text { Poland }\end{array}$ & 47.67 & 95.81 & 2.21 \\
$\begin{array}{l}\text { Boulder (BOU), } \\
\text { Colorado, US }\end{array}$ & 48.69 & 321.38 & 2.29 \\
$\begin{array}{l}\text { Fredericksburg } \\
\text { (FRD), Virginia, US }\end{array}$ & 48.05 & 359.38 & 2.24 \\
$\begin{array}{l}\text { Grabnik (GRB), } \\
\text { Poland }\end{array}$ & 46.28 & 97.83 & 2.13 \\
$\begin{array}{l}\text { Kiev (KIV), } \\
\text { Ukraine }\end{array}$ & 46.67 & 104.11 & 2.12 \\
$\begin{array}{l}\text { Mbour (MBO), } \\
\text { Senegal }\end{array}$ & 3.16 & 57.87. & - \\
$\begin{array}{l}\text { Odessa (ODE), } \\
\text { Ukraine }\end{array}$ & 42.28 & 104.14 & 1.86 \\
$\begin{array}{l}\text { Suwałki (SUW), } \\
\text { Poland }\end{array}$ & 50.10 & 98.58 & 2.47 \\
\hline
\end{tabular}

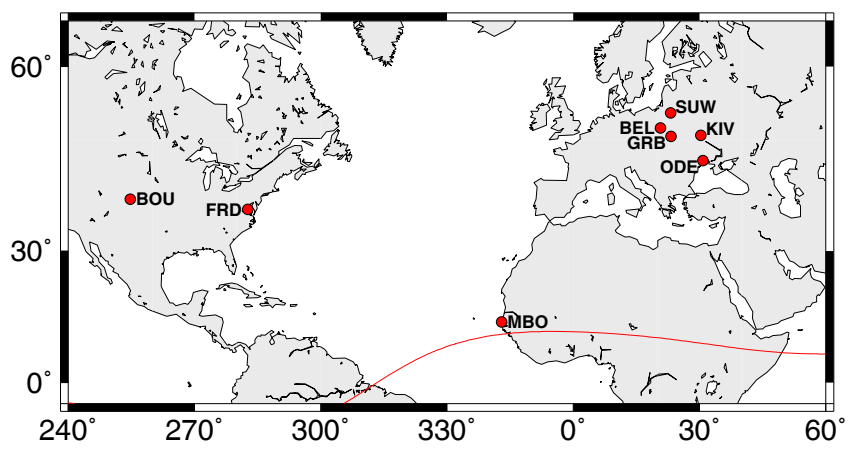

Figure 3. Map with site locations. The red line marks the magnetic Equator following Emmert et al. (2010).

$b$ controls the shifting of the wavelet along the signal and ensures that the information on frequency content of a given coefficient can be assigned to a particular time. This is important when analyzing non-stationary processes or looking for rarely occurring phenomena, and it constitutes the main advantage of the wavelet transform over the Fourier transform $\mathcal{F}$.

Certain properties of the wavelet transform allow for a re-consideration of Eq. (1) that can save much computing power. First, according to the convolution theorem, for a constant $a, W_{\mathrm{H}}$ can be regarded as the inverse Fourier transform of the product of the Fourier transforms of signal $H$ and wavelet $\Psi$ :

$W_{\mathrm{H}}=\mathcal{F}^{-1}\{\mathcal{F}(H) \mathcal{F}(\Psi)\}$.

Second, $(\mathcal{F}(\Psi))$ has specific properties. Its Fourier spectrum is narrow and self-similar under dilatation. This means that if we are not interested in a very fine frequency resolution and 
consider only $a$ values differing by a constant factor (typically 2), $\mathcal{F}(\Psi)$ has to be calculated only for one $a$ and for the rest $\mathcal{F}(\Psi)$ can be reconstructed, basically by shifting it through frequency space. Furthermore, the multiplication in Eq. (3) has to be carried out only where $(\mathcal{F}(\Psi) \neq 0)$. These considerations lead to the fast wavelet transform (cf., e.g., Blatter, 2003). Both versions, the latter and the one described in Eq. (1), have been applied in this study.

By means of the wavelet coefficients of synchronous signals of two observatories, e.g., $H_{1}$ and $H_{2}$, their coherence can be calculated according to

$\gamma^{2}(\omega)=\frac{\left|\left\langle H_{1}(\omega) H_{2}^{*}(\omega)\right\rangle\right|^{2}}{\left\langle H_{1}(\omega) H_{1}^{*}(\omega)\right\rangle\left\langle H_{2}(\omega) H_{2}^{*}(\omega)\right\rangle}$.

The relation between $a$, period $T$, and angular frequency $\omega$ is

$T=a \sqrt{2 \ln (2)}=\frac{2 \pi}{\omega}$.

Brackets \langle\rangle indicate a stacking over a certain number of coefficients at subsequent $b$ values.

\subsection{Transfer functions}

When carrying out induction soundings, the area for which a model of electric resistivity shall be derived is covered with a profile or an array of stations which measure the variations of magnetic $(X, Y, Z)$ and, in the case of MT, electric $\left(E_{x}\right.$, $\left.E_{y}\right)$ field components. For an LMT campaign each measurement lasts usually $2-3$ weeks and the sampling interval is $1 \mathrm{~s}$ or longer. During the data processing transfer functions between these components are derived which are introduced in the following. All of them are complex functions of frequency $\omega$. Usually they vary only smoothly over frequency (or period), and their real and imaginary parts (or absolute values and phases) are connected with each other in a relationship that is typical of induction processes.

GDS is of special interest here because geomagnetic observation data can be utilized. Here a two-component transfer function $(A(\omega), B(\omega))$ between a station's $Z$ component on the one hand and the $X$ and $Y$ components on the other hand is estimated which satisfies the equation

$Z(\omega)=A(\omega) X(\omega)+B(\omega) Y(\omega)$.

$(A(\omega), B(\omega))$ is usually displayed as induction arrows (a real and an imaginary one) with $A$ as north and $B$ as the east component as in Fig. 1. The solution of Eq. (6) for $(A(\omega), B(\omega))$ in terms of least squares is

$(A, B)=\left\{(\vec{X}, \vec{Y})^{\dagger}(\vec{X}, \vec{Y})\right\}^{-1}\left\{\vec{Z}^{\dagger}(\vec{X}, \vec{Y})\right\}$,

where the vectors consist of a large number $N$ of coefficients at the same frequency, e.g., $\vec{X}(\omega)=$
$\left(X_{1}(\omega), X_{2}(\omega), \ldots, X_{N}(\omega)\right)^{\top} . \mathrm{A}^{\top}$ denotes the transpose and $\mathrm{a}^{\dagger}$ the Hermitian transpose.

In MT the magnetotelluric transfer function between the horizontal electric and horizontal magnetic components of a station is calculated. It has the shape of a $2 \times 2$ tensor $\mathbf{Z}(\omega)=\left(\left(Z_{x x}(\omega), Z_{y x}(\omega)\right)^{\top},\left(Z_{x y}(\omega), Z_{y y}(\omega)\right)^{\top}\right)$ and satisfies the equation

$\left(E_{x}(\omega), E_{y}(\omega)\right)^{\top}=\mathbf{Z}(X(\omega), Y(\omega))^{\top}$.

The solution for $\mathbf{Z}(\omega)$ is

$\mathbf{Z}^{\top}=\left\{(\vec{X}, \vec{Y})^{\dagger}(\vec{X}, \vec{Y})\right\}^{-1}\left\{\left(\overrightarrow{E_{x}}, \overrightarrow{E_{y}}\right)^{\dagger}(\vec{X}, \vec{Y})\right\}$

$\mathbf{Z}$ is displayed as so-called sounding curves consisting of apparent resistivy $\rho_{\mathrm{a}}\left(\right.$ e.g., $\left.\rho_{\mathrm{a}}^{x y}=\mu_{0} /(2 \pi)\left|Z_{x y}\right|^{2} T\right)$ and phases $\phi_{x y}=\arctan \left(\mathfrak{I m} Z_{x y} / \mathfrak{R e} Z_{x y}\right)$. In case of not too complicated resistivity structures the off-diagonal elements $Z_{x y}$, $Z_{y x}$ are much larger than the others; thus, often only they are presented; cf. Fig. $2 \mathrm{~b}$ and c.

The solutions for $(A, B)$ and $\mathbf{Z}$ can become biased and unusable in the presence of certain types of anthropogenic noise. A crass example is the electromagnetic signals transmitted from DC railways, since they spread over distances of ca. $100 \mathrm{~km}$ from the source and are contradictory to the plane-wave assumption. A remedy for this is the remotereference method. This means that data $\overrightarrow{X_{R}}, \overrightarrow{Y_{R}}$ are included in the processing which have been measured at another variometer station that is sufficiently remote for the noise to be damped away, whereas the natural signal remains correlated. In many cases it is practical to use observatories as remotereference stations. Thereby, e.g., Eq. (9) changes to

$\mathbf{Z}_{\mathrm{RR}}^{\top}=\left\{(\vec{X}, \vec{Y})^{\dagger}\left(\overrightarrow{X_{\mathrm{R}}}, \overrightarrow{Y_{\mathrm{R}}}\right)\right\}^{-1}\left\{\left(\overrightarrow{E_{x}}, \overrightarrow{E_{y}}\right)^{\dagger}\left(\overrightarrow{X_{\mathrm{R}}}, \overrightarrow{Y_{\mathrm{R}}}\right)\right\}$

This leads to a stacking over cross spectra (instead of auto spectra in Eq. 7) which suppresses noise features that are incoherent between both sites. The usage of remote data in both enumerator and denominator warrants that the information content remains unchanged compared to Eq. (9). The approach in Eq. (9) is referred to as the single-site solution to distinguish it from the remote-reference approach (Eq. 10).

If the plane-wave assumption is taken seriously, the following argumentation is possible: the incident magnetic field at two stations of an array is exactly equal. Then differences between stations come only from the much smaller secondary magnetic fields which accompany currents locally induced in the conducting solid earth by the incident field. Therefore the inter-station transfer function HMT = $\left(\left(\mathrm{HMT}_{x x}, \mathrm{HMT}_{y x}\right)^{\top},\left(\mathrm{HMT}_{x y}, \mathrm{HMT}_{y y}\right)^{\top}\right)$ between the horizontal magnetic components of two stations

$(X, Y)^{\top}=\mathbf{H M T}\left(X_{\mathrm{RR}}, Y_{\mathrm{RR}}\right)^{\top}$

only gently deviates from the unity matrix and is indicative of induction and conductivity distribution. This is the idea 
of magnetovariational sounding, where inter-station transfer functions serve as input data for a modeling of the resistivity distribution in the subsurface. It has already been mentioned that the HMT displayed in Fig. 2a does not meet this expectation for part of the period range.

It can be shown (Schmucker, 1984; Neska, 2006; Campanyà et al., 2014) that the remote-reference solution for MT (Eq. 10) and GDS transfer functions can be formulated in a way that makes use of the inter-station transfer function. Thereby a quasi-magnetotelluric transfer function $Z^{q}$ is calculated between horizontal electric components of one station and horizontal magnetic components of another (reference) station, which is then multiplied by the inverse of the inter-station transfer function between the same stations:

$\mathbf{Z}_{\mathrm{RR}}^{\top}=\left(\mathbf{H} \mathbf{M} \mathbf{T}^{\top}\right)^{-1} \mathbf{Z}^{q^{\top}}$.

For the estimation of most transfer functions within this study, Egbert's code (Egbert and Booker, 1986) has been used. It applies, like all modern processing codes in this domain, robust instead of least-square statistics to stabilize results against outliers in the data. To finish this section we provide the formula for the skin depth $\delta$. For the simplest model of resistivity distribution in the solid earth, a homogeneous halfspace of resistivity $\rho$ (in $\Omega \mathrm{m}$ ), the skin depth in $\mathrm{km}$ amounts to

$\delta \approx \frac{1}{2} \sqrt{\rho T}$

\section{Results and discussion}

Figure 4 shows dynamic amplitude spectra and mutual coherences for $H$ components of the KIV, MBO, and BOU observatories during 1 day in March 2013. The amplitude spectra have been obtained via the "slow" wavelet transform (Eq. 1); for this reason the resolution over period is better. The time resolution, i.e., the interval from which coefficients were taken for one stacked value, is about $10 \mathrm{~min}$, with small differences from period to period. The coherences come from the fast wavelet transform (Eq. 3) and the time resolution is $15 \mathrm{~min}$. Thereby one coherence value for $100 \mathrm{~s}$ is stacked over 85 coefficients, for $50 \mathrm{~s}$ over 170 , and for $25 \mathrm{~s}$ over 341 ones. Although maximum activity takes place around local noon and is therefore shifted between observatories, a number of striking events in the spectra happen synchronously in all observatories and lead not throughout but often to medium or high coherence values. That the signals behind such values are really pulsations is frequently very obvious in time series, too; cf. Fig. 5. From both pictures it is evident that there exist pulsations that cannot originate from field line resonances since they are present at the Equator (far from footpoints of field lines) and have a quasi-global coherence length. If one looks up the literature for a possible origin
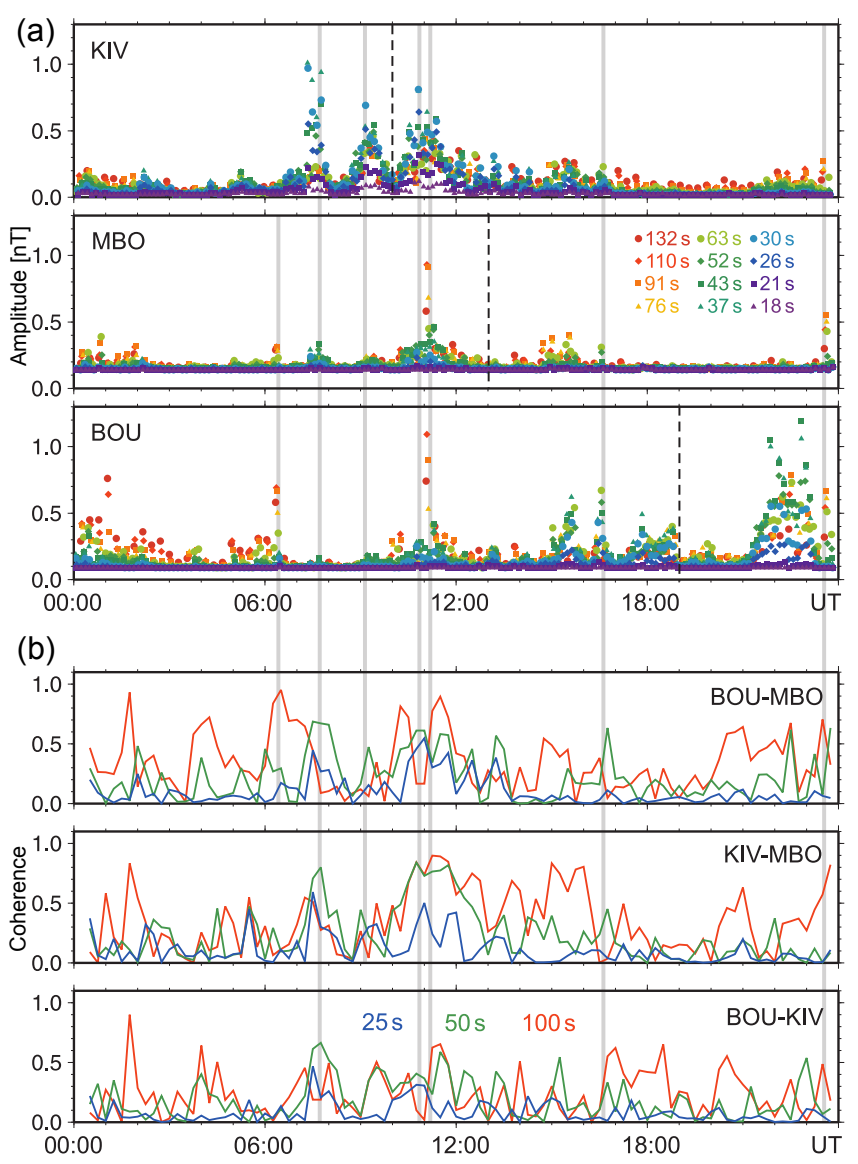

Figure 4. Dynamic amplitude spectra of (a) and coherences between (b) $H$ components of $\mathrm{KIV}, \mathrm{MBO}$, and BOU observatories for 4 March 2013, for a number of pc3-4 periods. Although the main activity takes place around local noon (marked with vertical dashed lines in a), many events take place synchronously in these distant places, and often short-term coherence (time resolution: $15 \mathrm{~min}$ ) is not low. Such events are marked with grey vertical lines.

of pulsations with such properties, one gets a clear answer, at least for the pc3 and pc4 range. These are most probably upstream waves. The following description of their generation follows mainly but not only Heilig et al. (2007).

In the terrestrial foreshock region ions originating from solar wind and having been deflected from the terrestrial magnetosphere are subjected to cyclotronic resonance in the interplanetary magnetic field. Thereby they produce a signal at the cyclotron resonant frequency that propagates upstream from the solar wind. But since the velocity of the latter is greater than the propagation velocity of the former, these upstream waves are swept back against the magnetopause and, under convenient conditions, couple to the magnetosphere. Then they propagate in the form of compressional waves (fast Alfvénic mode) partly directly through the magnetosphere. Where they encounter a magnetic field line with a component parallel to their oscillation direction, they couple to it, and if its resonant frequency matches their own frequency, 


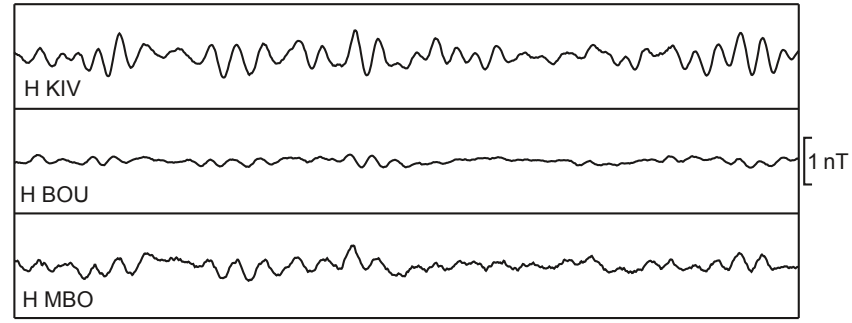

19 min on 20 March 2013, 11:19:32 UT

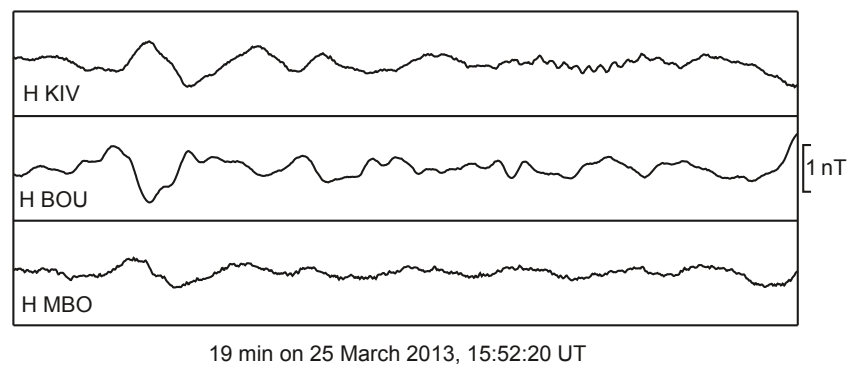

Figure 5. Two examples of highly coherent synchronous time series between KIV, BOU, and MBO $H$ components. The upper part shows pulsations in the $\mathrm{pc} 3$ range which originate most probably from upstream waves. The example belows contains pc5 pulsations to the left, probably from another global source mechanism. The time series are high-pass filtered with a cut-off period of $300 \mathrm{~s}$.

they excite field line resonances which have a different oscillation direction. In the ionosphere all ULF waves experience a rotation of their polarization (e.g., Vellante et al., 2004), such that field line resonances and direct upstream waves cannot be distinguished easily in the geomagnetic pulsations as which they are observable on the ground. Data from the interplanetary magnetic field enable identification of upstream waves since there is a (in first approximation) simple linear relationship between its strength and their frequency (e.g., Verõ et al., 1998; Howard and Menk, 2005; Heilig et al., 2007; Yagova et al., 2017). Multi-spacecraft data led also to the result that the coherence length of upstream waves in the foreshock region is on the order of magnitude of 1 earth radius (Le and Russell, 1990). Comparison of spacecraft data mapping the inner magnetosphere with a mid latitude ground station revealed a practical dayside-wide coherence of upstream waves in geomagnetic low and mid latitudes (Heilig et al., 2007). The same study confirms a maximum of upstream wave power in the equatorial pre-noon region. Upstream waves can also be refracted into the nightside of the magnetosphere and then have smaller amplitudes (Yagova et al., 2017); this effect is visible for BOU in the upper part of Fig. 5.

Pulsations in the region of the geomagnetic Equator are special. They possess practically no $D$ component and the amplitudes of the $H$ component are larger than at the adjacent low latitudes. This phenomenon is referred to as the equatorial enhancement. It is not clear whether this is the rea-
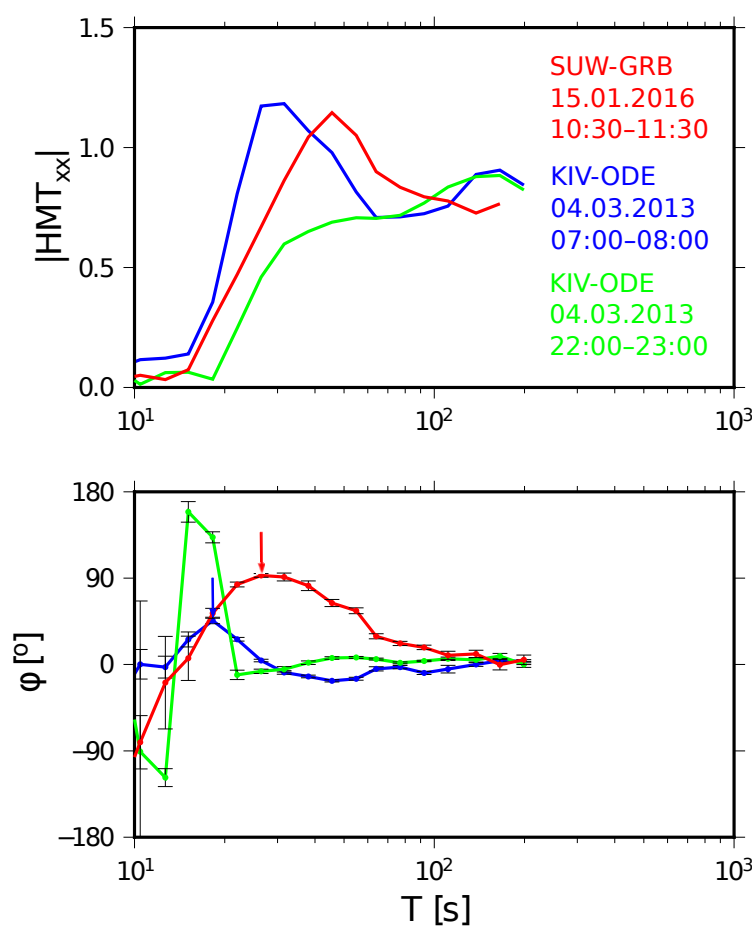

Figure 6. $x x$ element of the inter-station transfer function between SUW and GRB (red) and KIV and ODE (blue) for $1 \mathrm{~h}$ of data. Both show the typical pattern for field line resonances that allows for determination of the resonant frequency (indicated by arrows). It is $18 \mathrm{~s}(55 \mathrm{mHz})$ for the KIV-ODE pairing (midpoint latitude $44.5^{\circ}$ ) and $26 \mathrm{~s}(37 \mathrm{mHz})$ for SUW-GRB $\left(48.2^{\circ}\right)$. The green curve representing KIV-ODE for another hour of data demonstrates that this transfer function is not stable in time, and such features may not reliably appear because of a temporal lack of such resonances (almost zero absolute value below $20 \mathrm{~s}$ ).

son for difficulties in equatorial LMT. Similarly, the reason for the enhancement itself is not clear. Proposed explanations comprise both interactions with the electrojet and the idea that the geometry of the magnetosphere causes the equatorial plane to be less dissipative for waves traveling through it (Yagova et al., 2017, and citations therein).

Ground-based research beyond low latitudes confirms that field line resonances are excited by upstream waves and that the latter are not less common than the former (Verõ et al., 1998; Howard and Menk, 2005). They also report pulsation events coherent over thousands of kilometers (Verõ et al., 1998; Yagova et al., 2017). Analysis tools in this domain are extraordinarily fine compared to MT ones; frequency resolution can reach a few millihertz and in time structures can be caught that exist only for some minutes as shown in Verõ et al. (1998). These authors also report that structures typical for upstream waves and structures typical for field line resonances alternate rapidly and in a way that suggests that upstream waves initiate and finish field line resonances. Identification of field line resonances is, beside other tech- 

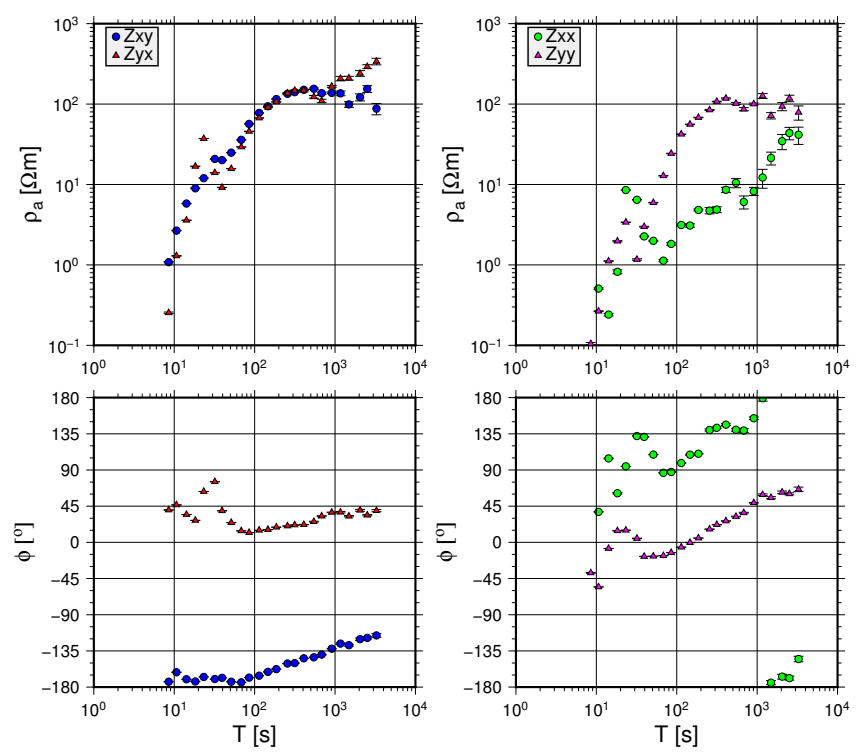

Figure 7. Sounding curves from the quasi-magnetotelluric transfer function between electric components of GRB and horizontal magnetic ones of SUW. Data are the same as in Fig. 2. Note the similarity to the inter-station transfer function (Fig. 2a) in the "erratic" short-period behavior.

niques, possible by means of two longitudinally arranged stations spaced by at least $80-100 \mathrm{~km}$ in distance, which is the estimated surface width of resonant shells (Waters et al., 1991; Verõ et al., 1998). The technique makes use of the fact that a signal resulting from the superposition of two (monochromatic) signals with nearly but not completely the same frequency exhibits a minimum-maximum transition in amplitude and a maximum in phase at the midpoint between both frequencies (Waters et al., 1991). It is called the cross-phase technique and applied not only for verification of field line resonances, but also for determination of their resonant frequency (e.g., Vellante et al., 2004; Lichtenberger et al., 2013). Interestingly, its implementation is formally almost equal to an inter-station transfer function (Egbert et al., 2000). Figure 6 shows $x x$ components of such a transfer function between two appropriate station pairs (SUW-GRB and KIV-ODE; cf. Fig. 3) obtained from $1 \mathrm{~h}$ of data and based on wavelet coefficients. The expected features are visible in the blue and red curves; according to the phase maxima the resonant frequencies amount to ca. $55 \mathrm{mHz}(18 \mathrm{~s})$ for KIVODE and ca. $37 \mathrm{mHz}(26 \mathrm{~s})$ for SUW-GRB. It meets expectations that the pairing at a higher latitude has a lower frequency. Comparison with the correspondent time in Fig. 4 hints at broadband activity in both and coherence between $\mathrm{KIV}$ and MBO. Since this cannot be explained by field line resonances alone, it is very probable that upstream waves stand behind the blue curve in Fig. 6 as well. The midpoint latitude of SUW-GRB is $48.2^{\circ}$, which is almost equal to the geomagnetic latitude of Niemegk observatory in 1985
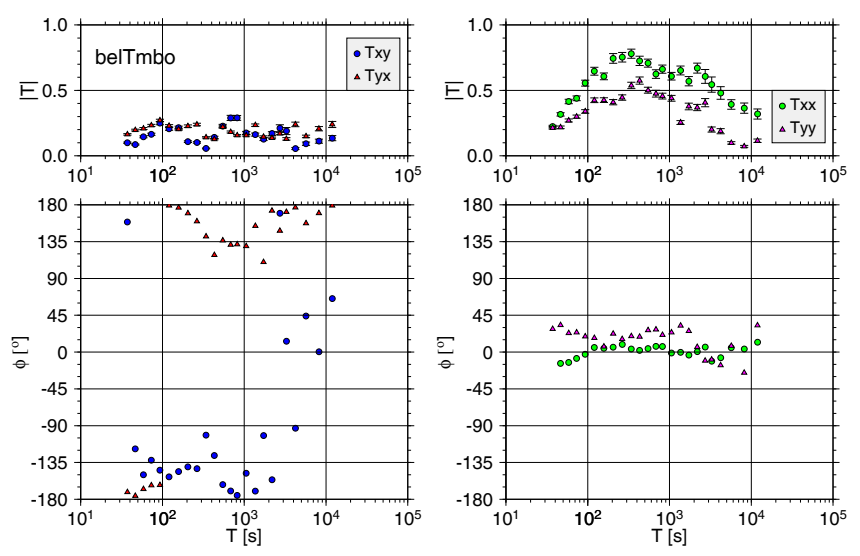

Figure 8. Inter-station transfer function between BEL (Poland) and MBO (Senegal) for 101 days of data.

$\left(48.0^{\circ}\right)$. Data from this place and time were analyzed by Vellante et al. (1993), who found that the resonant frequency amounts to $30-35 \mathrm{mHz}$. Given the poor frequency resolution in our method, we regard this as a confirmation of our result. The green curve in Fig. 6 has been obtained for another hour of KIV-ODE data. We interpret the scatter in phase and the missing maximum in amplitude as a sign of weak or absent field line resonances at this time. Comparison with Fig. 4 reveals that activity at the resonant frequency was absent in both KIV and MBO at this time. There is, however, some coherent activity at longer periods that could be possibly ascribed to upstream waves.

The result in Fig. 6 confronts us with two insights that may appear somewhat heretical from the point of view of induction soundings. Inter-station transfer functions can change in time (depending on the presence/absence of field line resonances), and they can have a physical meaning beyond electromagnetic induction (exhibiting a resonant frequency). In this light the inter-station transfer function in Fig. 2a does not appear meaningless. The putative distortions are simply a (even if coarsely resolved) signature of field line resonances. Obviously the violation of the postulate that the incident field at both stations must be equal prevents the inter-station transfer function from taking a shape interpretable for MV. The question why MT works for this example can be answered in two ways. The first one is formal. Figure 7 shows $Z^{q}$ for these data which exhibits analogous resonance signatures. Application of Eq. (12) means, roughly speaking, that it is divided by the HMT in Fig. 2a. Thereby resonance features are cancelled out and a flawless RR result remains (Fig. 2c). The second way consists in taking the postulates seriously. The plane-wave assumption for local (not inter-station) transfer functions does not mean that the incident waves must be truly plane. They have to be plane on a scale greater than the skin depth. If we take the width of a resonant shell as the scale of inhomogeneity and assume that field line resonances at mid latitudes are limited to the pc3 range (10- 
$45 \mathrm{~s}$ ), application of Eq. (13) reveals that up to a halfspace resistivity $\sim 500 \Omega \mathrm{m}$ field line resonances are not an obstacle for MT and GDS. This is confirmed by Pilipenko and Fedorov (1993), who point out that only low-conductive structures can be affected by this problem. In cases where MT or GDS results in high-resistive regions are affected by field line resonances, it might be a good suggestion to attempt a remote-reference application of an equatorial station (which is free of such resonances). In this way at least resonances that do not occur at the same time as upstream waves would be prevented from entering the transfer functions.

An inter-station transfer function between mid latitudes and the Equator (corresponding to the third panel in Fig. 1) is shown in Fig. 8. As discussed above, an induction-based interpretation seems highly improbable. However, some parts of it are not too far from the unity matrix, e.g., the pc5 range $(150-600 \mathrm{~s})$ of the $x x$ element. This fits to the finding that long-term coherences for a similar station pairing in this range are not really low ( $>0.3$; see Table 1$)$.

There remains the apparent contradiction that for shorter periods, long-term coherences are very low (ibid.) but shortterm ones are not (Fig. 4b). This seems to be a systematic tendency. We have additionally calculated coherences for the whole of March 2013 during $4 \mathrm{~h}$ intervals. In general such pc3-4 coherences are low on an inter-continental scale, but for a few $4 \mathrm{~h}$ intervals during that month they are medium to high (not shown). Analogous traces of such a trend can be found in the literature. Ochadlick (1990) reports that $20 \mathrm{~min}$ coherence in airborne pulsations $\leqslant 25 \mathrm{~s}$ drops at a distance of $150 \mathrm{~km}$, whereas Heilig et al. (2007) find dayside-wide 1 min coherence for upstream waves. These findings could be a hint at a possible time dependency in far-distant interstation transfer functions in the pulsation range.

\section{Conclusions}

It has to be admitted that currently, it is difficult to understand what is state of the art in fundamental research on geomagnetic pulsations. That is because "The bibliography [...even on a section of this domain - insertion by the authors] includes several hundred publications" (Yagova et al., 2017) and because currently there is a large number of new insights in this area, but also a series of open questions (Menk, 2011) and therefore no clear, comprehensive, and consistent picture. Nevertheless it raises doubts that 20 years after their importance for geomagnetic pulsations was noticed, upstream waves are not mentioned in the signal source sections of magnetotelluric textbooks. Given the fact that due to their large coherence lengths these waves have very convenient properties for induction soundings, it seems inappropriate that many MT workers appear unaware of them, whereas the much more problematic field line resonances are relatively well represented in the MT literature.
The peculiarities of LMT sources in the magnetic equatorial region are currently not well understood, but are still under debate. This should be clearly pointed out instead of making vague references to the electrojet. Such a clarification would clear the way for unprejudiced research in this area. Research of this type appears promising, particularly with regard to equatorial stations as distant reference sites. This idea is justified by the findings that (a) equatorial pulsations are more directly ascribable to upstream waves and not "disturbed" by field line resonances, and (b) the equatorial region is privileged in terms of high large-distance coherence of upstream waves with (at least) mid latitude regions.

When considering the question whether the plane-wave assumption is violated by field line resonances, a clear discrimination between estimation of inter-station transfer functions (for MV sounding) and MT/GDS transfer functions has to be made. This is because the MV precondition that the incident field at both stations has to be equal is very strict. It is readily violated in everyday situations. A north-south spacing of $80-100 \mathrm{~km}$ is sufficient to produce effects around the resonant frequency in the inter-station transfer function that make it unusable for a sounding interpretation unless special MV processing schemes (which remove data fragments leading to non-stationary results far from the unity matrix, Ritter et al., 1998) are applied. In contrast, the precondition for usable local transfer functions is weaker. It only says that the skin depth of the incident wave has to be significantly smaller than the mentioned distance. This is the case at mid latitudes for low to moderate resistivities of the subsurface and only massive high-resistive structures give reason for caution. In other words, situations may be encountered where MT/GDS is well possible in the pulsation range although MV (with the same data) is not. RR can be applied with such data, and it is not a contradiction that RR can be formulated in a way that relies on the inter-station transfer function. This is because $\mathrm{RR}$ does not require that the inter-station transfer function makes sense in the MV paradigm; it seems to be sufficient that it exists. The latter sentence is - together with the insight that many LMT sources have a global rather than local origin - also the key to the understanding that reference sites may be situated at much larger distances than assumed so far.

Code and data availability. Data from BEL, BOU, FRD, and MBO are available at the INTERMAGNET database. Data from SUW used for Fig. 1 and from KIV and ODE have been added to this publication as supplement. Data from SUW used in Figs. 2, 6, and 7 and from GRB are part of a running project and therefore are not available before the project ends in summer 2018. They may become available at a later time on request to Vladimir Semenov (sem@igf.edu.pl) or Mariusz Neska (nemar@igf.edu.pl). The MT processing code by Gary Egbert is available, see reference list (Egbert and Booker, 1986). All other codes used in this study are available on request to Anne Neska (anne@igf.edu.pl). 


\section{The Supplement related to this article is available online at https://doi.org/10.5194/angeo-36-337-2018- supplement.}

Competing interests. The authors declare that they have no conflict of interest.

Special issue statement. This article is part of the special issue "The Earth's magnetic field: measurements, data, and applications from ground observations (ANGEO/GI inter-journal SI)". It is a result of the XVIIth IAGA Workshop on Geomagnetic Observatory Instruments, Data Acquisition and Processing, Dourbes, Belgium, 4-10 September 2016.

Acknowledgements. The results presented in this paper rely on data collected at magnetic observatories. We thank the national institutes that support them and INTERMAGNET for promoting high standards of magnetic observatory practice (www. intermagnet.org). This work was supported within statutory activities no. 3841/E-41/S/2017 and relies on data from grant no. NCN 2014/15/B/ST10/00789 of the Ministry of Science and Higher Education of Poland. We thank the topical editor Arnaud Chulliat for handling the manuscript and the reviewers Frederick Menk and Andreas Junge for many invaluable hints that helped to improve it.

The topical editor, Arnaud Chulliat, thanks Frederick Menk and one anonymous referee for help in evaluating this paper.

\section{References}

Bahr, K. and Simpson, F.: Practical magnetotellurics, Cambridge University Press, Cambridge, 2005.

Balasis, G., Maus, S., Lühr, H., and Rother, M.: Wavelet Analysis of CHAMP Flux Gate Magnetometer Data, Springer, Berlin, Heidelberg, 347-352, https://doi.org/10.1007/3-540-26800-6_55, 2005.

Balasis, G., Daglis, I. A., Georgiou, M., Papadimitriou, C., and Haagmans, R.: Magnetospheric ULF wave studies in the frame of Swarm mission: a time-frequency analysis tool for automated detection of pulsations in magnetic and electric field observations, Earth Planet. Space, 65, 1385-1398, https://doi.org/10.5047/eps.2013.10.003, 2013.

Blatter, C.: Wavelets - Eine Einführung, Advanced Lectures in Mathematics, Vieweg+Teubner Verlag, https://books.google.pl/ books?id=XCTwqrBbWQUC, 2003.

Campanyà, J., Ledo, J., Queralt, P., Marcuello, A., and Jones, A. G.: A new methodology to estimate magnetotelluric (MT) tensor relationships: Estimation of Local transfer-functIons by Combining Interstation Transfer-functions (ELICIT), Geophys. J. Int., 198, 484-494, https://doi.org/10.1093/gji/ggu147, 2014.

Chernogor, L. F.: Geomagnetic field fluctuations near Kharkov, which accompanied rocket launches from the Baikonur site, Geomagn. Aeronomy, 49, 384-396, https://doi.org/10.1134/S001679320903013X, 2009.
Cladis, J. B.: Multiply coupled oscillations of field lines in the magnetosphere: Modulation of trapped particles and ionospheric currents, J. Geophys. Res., 76, 2345-2356, https://doi.org/10.1029/JA076i010p02345, 1971.

Egbert, G. D. and Booker, J. R.: Robust estimation of geomagnetic transfer functions, code available at: http: //www.complete-mt-solutions.com/mtnet/programs/egbert.html, Geophys. J. Int., 87, 173-194, https://doi.org/10.1111/j.1365246X.1986.tb04552.x, 1986.

Egbert, G. D., Eisel, M., Boyd, O. S., and Morrison, H. F.: DC trains and Pc3s: Source effects in mid-latitude geomagnetic transfer functions, Geophys. Res. Lett., 27, 25-28, https://doi.org/10.1029/1999GL008369, 2000.

Emmert, J. T., Richmond, A. D., and Drob, D. P.: A computationally compact representation of Magnetic-Apex and Quasi-Dipole coordinates with smooth base vectors, J. Geophys. Res., 115, A08322, https://doi.org/10.1029/2010JA015326, 2010.

Garcia, X. and Jones, A. G.: Robust processing of magnetotelluric data in the AMT dead band using the continuous wavelet transform, Geophysics, 73, F223-F234, https://doi.org/10.1190/1.2987375, 2008.

Heilig, B., Lühr, H., and Rother, M.: Comprehensive study of ULF upstream waves observed in the topside ionosphere by CHAMP and on the ground, Ann. Geophys., 25, 737-754, 2007.

Houpt, L.: Neue elektromagnetische Untersuchungen zur norddeutschen Leitfähigkeitsanomalie, Diplomarbeit, Fachbereich Physik an der Technischen Universität Berlin, 2008.

Howard, T. A. and Menk, F. W.: Ground observations of highlatitude Pc3-4 ULF waves, J. Geophys. Res., 110, A04205, https://doi.org/10.1029/2004JA010417, 2005.

Jankowski, J. and Sucksdorff, C.: IAGA Guide for Magnetic Measurements and Observatory Practice, International Association of Geomagnetism and Aeronomy, Warsaw, https://books.google.pl/ books?id=UCM8PQAACAAJ, 1996.

Larsen, J. C., Mackie, R. L., Manzella, A., Fiordelisi, A., and Rieven, S.: Robust Smooth Magnetotelluric Transfer Functions, J. Geophys. Res., 124, 801-819, 1996.

Le, G. and Russell, C. T.: A study of the coherence length of ULF waves in the Earth's foreshock, J. Geophys. Res., 95, 1070310706, https://doi.org/10.1029/JA095iA07p10703, 1990.

Lichtenberger, J., Clilverd, M. A., Heilig, B., Vellante, M., Manninen, J., Rodger, C. J., Collier, A. B., Jorgensen, A. M., Reda, J., Holzworth, R. H., Friedel, R., and Simon-Wedlund, M.: The plasmasphere during a space weather event: first results from the PLASMON project, J. Space Weather Space Clim., 3, A23, https://doi.org/10.1051/swsc/2013045, 2013.

McIlwain, C. E.: Coordinates for mapping the distribution of magnetically trapped particles, J. Geophys. Res., 66, 3681-3691, https://doi.org/10.1029/JZ066i011p03681, 1961.

McPherron, R. L.: Magnetic Pulsations: Their Sources and Relationto Solar Wind and Geomagnetic Activity, Surv. Geophys., 26, 545-592, https://doi.org/10.1007/s10712-005-1758-7, 2005.

Menk, F. W.: Magnetospheric ULF Waves: A Review, Springer Netherlands, Dordrecht, 223-256, https://doi.org/10.1007/97894-007-0501-2_13, 2011.

Neska, A.: Remote Reference versus Signal-Noise Separation: A least-square based comparison between magnetotelluric processing techniques, PhD thesis, Institut für Geologische Wissenschaften, Freie Universität Berlin, 2006. 
Neska, A., Reda, J., Neska, M., and Sumaruk, Y.: On the influence of DC railway noise on variation data from Belsk and Lviv geomagnetic observatories, Acta Geophys., 61, 385-403, https://doi.org/10.2478/s11600-012-0058-0, 2013.

Ochadlick, A. R.: Time series and correlation of pulsations observed simultaneously by two aircraft, Geophys. Res. Lett., 17, 18891892, https://doi.org/10.1029/GL017i011p01889, 1990.

Padilha, A. L., Alves, A. R., Silva, G. D. B., and Espinoza, K. V.: Effect of a huge crustal conductivity anomaly on the H-component of geomagnetic variations recorded in central South America, Earth Planet. Space, 69, 2017.

Padua, M. B., Padilha, A. L., and Vitorello, I.: Disturbances on magnetotelluric data due to DC electrified railway: A case study from southeastern Brazil, Earth Planet. Space, 54, 591-596, 2002.

Pilipenko, V. A. and Fedorov, E. N.: Magnetotelluric sounding of the crust and hydromagnetic monitoring of the magnetosphere with the use of ULF waves, Ann. Geofis., 36, 19-31, https://doi.org/10.4401/ag-4243, 1993.

Ritter, O., Junge, A., and Dawes, G.: New equipment and processing for magnetotelluric remote reference observations, Geophys. J. Int., 132, 535-548, https://doi.org/10.1046/j.1365246X.1998.00440.x, 1998.

Schmucker, U.: EM Übertragungsfunktionen aus Beobachtungen mit mehreren gleichzeitig registrierenden Stationen, in: 10. Kolloquium Elektromagnetische Tiefenforschung, Grafrath in Oberbayern, 35-36, 1984.

Schäfer, A., Houpt, L., Brasse, H., and Hoffmann, N.: The North German Conductivity Anomaly revisited, Geophys. J. Int., 187, 85-98, https://doi.org/10.1111/j.1365-246X.2011.05145.x, 2011.

Turbitt, C., Matzka, J., Rasson, J., St-Louis, B., and Stewart, D.: An instrument performance and data quality standard for INTERMAGNET one-second data exchange, in: Proceedings of the XVth IAGA Workshop on Geomagnetic Observatory Instruments, Data Acquisition, and Processing, edited by: Hejda, P., Chulliat, A., and Catalán, M., 186-188, 2013.

Vassal, J., Menvielle, M., Cohen, Y., Dukhan, M., Doumouya, V., Boka, K., and Fambitakoye, O.: A study of transient variations in the Earth's electromagnetic field at equatorial electrojet latitudes in western Africa (Mali and the Ivory Coast), Ann. Geophys., 16, 677-697, 1998.
Vellante, M., Villante, U., Core, R., Best, A., Lenners, D., and Pilipenko, V. A.: Simultaneous geomagnetic pulsation observations at two latitudes - Resonant mode characteristics, Ann. Geophys., 11, 734-741, 1993.

Vellante, M., Lühr, H., Zhang, T. L., Wesztergom, V., Villante, U., De Lauretis, M., Piancatelli, A., Rother, M., Schwingenschuh, K., Koren, W., and Magnes, W.: Ground/satellite signatures of field line resonance: A test of theoretical predictions, J. Geophys. Res., 109, A06210, https://doi.org/10.1029/2004JA010392, 2004.

Verõ, J., Lühr, H., Vellante, M., Best, I., Střeštik, J., Miletits, J. C., Holló, L., Szendrõi, J., and Zieger, B.: Upstream waves and field line resonances: simultaneous presence and alternation in Pc3 pulsation events, Ann. Geophys., 16, 34-48, https://doi.org/10.1007/s00585-997-0034-1, 1998.

Viljanen, A.: Description of the magnetospheric/ionospheric sources, Cambridge University Press, https://doi.org/10.1017/CBO9781139020138.005, 2012.

Waters, C. L., Menk, F. W., and Fraser, B. J.: The resonance structure of low latitude Pc3 geomagnetic pulsations, Geophys. Res. Lett., 18, 2293-2296, https://doi.org/10.1029/91GL02550, 1991.

Yagova, N. V., Heilig, B., Pilipenko, V. A., Yoshikawa, A., Nosikova, N. S., Yumoto, K., and Reda, J.: Nighttime Pc3 pulsations: MM100 and MAGDAS observations, Earth Planet. Space, 69, https://doi.org/10.1186/s40623-017-0647-x, 2017. 